Abstract THU0605 - Table 1. Comparison of overall costs between persistent and non-persistent patients $(n=1,575)$

\begin{tabular}{|c|c|c|c|c|}
\hline & \multicolumn{3}{|c|}{ Mean cost/patient (SD) } & \multirow[t]{2}{*}{ P-value } \\
\hline & Persistent & Non-persistent & Difference (persistent - Non persistent) & \\
\hline Drugs costs & $10,726.29(6,285.74)$ & $7,443.47(10,199.31)$ & $3,282.82(-3,913.57)$ & $<0.0001$ \\
\hline Biotherapies & $9,520.45(3,601.16)$ & $3,807.88(4,887.48)$ & $5,712.57(-1,286.32)$ & $<0.0001$ \\
\hline Others & $1,205.84(5,596.59)$ & $3,635.59(9,818.49)$ & $-2,429.75(-4,221.89)$ & $<0.0001$ \\
\hline Non-drugs costs & $3,066.42(4,765.38)$ & $4,658.24(6,330.43)$ & $-1,591.81(-1,565.04)$ & $<0.0001$ \\
\hline Including sick leave compensations & $525.00(2,029.22)$ & $1,162.55(3,309.37)$ & $-637.55(-1,280.15)$ & $<0.0001$ \\
\hline Hospital admissions & $1,234.64(3,957.32)$ & $3,712.15(7,124.84)$ & $-2,477.50(-3,167.52)$ & $<0.0001$ \\
\hline Total cost & $15,027.36(10,268.24)$ & $15,813.86(16,976.21)$ & $-786.50(-6,707.97)$ & 0.0495 \\
\hline
\end{tabular}

estimated with Kaplan Meier analysis. A patient was considered as non-persistent in the event of a prolonged interruption of the therapy during 91 days or more. Persistent and non-persistent patients were compared, after a 1:1 propensity score matching, in term of medical resource utilization and costs (from a National Health Service perspective) in the subsequent 12-month period.

Results: Among 3,804 patients initiating treatment with an SC-TNFi in France between 2012/07/01 and 2012/12/31, 2,133 were classified as persistent at 12 months and 1,671 as non-persistent. After the 1:1 propensity score matching, 1,575 patients were studied in each group. Persistent patients had a lower overall cost than non-persistent patients $(-787 €)$ : persistent patients had higher costs for drugs $(+3,283 €)$, due to the cost of biotherapies, but had lower costs associated to non-drugs $(-1,592 €)$ and hospital admissions $(-2,478 €)$.

Conclusions: The results indicate that persistence to treatment with SC-TNFi may be associated with cost offsets in terms of non-biologic costs, particularly for hospital admissions. However, as always the case with observational data, residual confounding factors could explain part of the results (e.g. disease severity).

Disclosure of Interest: M. Belhassen Employee of: PELyon, F. Tubach: None declared, C. Hudry Consultant for: ABBVIE, MSD, BMS, PFIZER, ROCHE, CELGENE, NOVARTIS, BIOGEN, UCB, SANDOZ, AMGEN., Employee of: COCHIN Hospital, M.-C. Woronoff: None declared, L. Levy-Bachelot Employee of: MSD France, L. Lamezec Employee of: MSD France, E. Van Ganse Consultant for: PELyon; ALK ABELLO; AstraZeneca; Bayer; BMS; BIF; GSK; IMS; LASER; MSD, B. Fautrel Grant/research support from: AbbVie, MSD, Pfizer, Consultant for: AbbVie, Biogen, BMS, Celgène, Hospira, Janssen, Lilly, MSD, NORDIC Pharma, Pfizer, Roche, SOBI, UCB

DOI: 10.1136/annrheumdis-2017-eular.2129

\section{THU0606 COGNITIVE DYSFUNCTION IN CONNECTIVE TISSUE DISEASES}

A. Dima ${ }^{1}$, L. Groseanu ${ }^{1,2}$, A. Balanescu ${ }^{1,2}$, D. Predeteanu ${ }^{1,2}$

D. Opris-Belinski ${ }^{1,2}$, A. Borangiu ${ }^{1,2}$, D. Mazilu ${ }^{1,2}$, V. Bojinca ${ }^{1,2}$, R. Ionescu ${ }^{1,2}$.

${ }^{1}$ Internal Medicine and Rheumatology, Sf Maria Clinical Hospital; ${ }^{2}$ Department 5 - Internal Medicine, University of Medicine and Pharmacy Carol Davila,

Bucharest, Romania

Background: Several studies documented the presence of cognitive dysfunction in different rheumatologic autoimmune diseases, but the absence of standard criteria of diagnostic and of an index with patients in which this dysfunction occurs, makes the mentioned studies often lead to divergent conclusions.

Objectives: To evaluate the impact of four autoimmune diseases:Systemic Lupus Erythematosus (SLE), Rheumatoid Arthritis (RA), Systemic Sclerosis (SSc) and Ankylosing Spondylitis (AS) on patient cognition and to identify potential factors which lead to cognitive dysfunction occurrence in this diseases.

Methods: This is a case-control study that included randomly selected patients with SLE, RA, SSc and AS from a University hospital and a matched control group. Data collected included: demographics, patients' education and specific data related to disease (duration, activity scores: DAS284v for RA patients, BASDAI in patients with AS, SLICC/SLEDAI for SLE patients and EUSTAR score/Rodnan score for SSc patients, damage indexes, organ involvement, treatment) and comorbidities. Cognition was assesed using MoCA Test (Montreal Cognitive Assessment The Test). The data were then processed using SPSS 23 version software.

Results: The study group included 255 patients: 58 RA patients, 52 SLE, 70 SSc and 25 AS patients and 50 healthy matched controls.

In all groups of patients, cognitive dysfunction prevalence was higher than control group (RA-64.98\%, SLE $-57.69 \%$, SS $-44.29 \%$, AS $-35.72 \%$ vs. $24 \%$ in the control group).

The differences were statistically significant for the RA group $(p=0.00)$, the SLE group ( $p=0.001)$, and the SS group $(p=0.001)$

For the RA group none of the items analyzed (demographics, disease characteristic, patient" education) showed a significant correlation with cognitive dyfunction. The same lack of correlation was also noted in AS and SSc patients. For the SLE group the only variable analyzed with a significant impact statistically was the SLEDAI score over $12(\mathrm{p}=0.002)$.

Also, this study did not find a significant statistically association between the presence of antiphospholipid syndrome.

Cognitive dysfunction seems to be more frequent and severe in RA and SLE patients compared to AS patients $(p=0.014$, respectively $p=0.05)$.

Conclusions: The results obtained in this study show that, indeed, cognitive dysfunction is an issue to be watched very carefully in patients with autoimmune diseases. The appearance of cognitive dysfunction has a negative impact on life quality of these patients, the pathophysiological mechanisms that contribute to it's appearance are intricate and difficult to isolate.

References:

[1] Sabbadini MG, Manfredi AA, Bozzolo E, et al. Central nervous system involvement in systemic lupus erythematosus patients without overt neuropsychiatric manifestations. Lupus 1999; 8: 11-19.

[2] L. Groseanu, T. Gudu, R. Ionescu. Significance of cognitive impairment in systemic sclerosis. Ann Rheum Dis2016;75(Suppl2): 526.

[3] Appenzeller S, Bertolo MB, Costallat LT. Cognitive impairment in rheumatoid arthritis. Methods Find Exp Clin Pharmacol 2004;26:339-43.

Disclosure of Interest: None declared

DOI: 10.1136/annrheumdis-2017-eular.5206

\section{THU0607 RISK FACTORS FOR EARLY RETIREMENT IN SYSTEMIC SCLEROSIS}

A.M. Gheorghiu $^{1,2}$, C. Vrancianu ${ }^{1,2}$, C. Draganescu ${ }^{1,2}$, R. Oneata ${ }^{1,2}$,

L. Macovei ${ }^{1,2}$, M. Sasu ${ }^{1,2}$, M. Milicescu ${ }^{1,2}$, O. Olteanu ${ }^{1,3}$, M. Speriatu ${ }^{1,2}$,

M. Bojincă ${ }^{1,2}$, V. Stoica ${ }^{1,2}$, C. Mihai ${ }^{1,2}$. ${ }^{1}$ Carol Davila University of Medicine and

Pharmacy; ${ }^{2}$ Internal Medicine and Rheumatology; ${ }^{3}$ Cantacuzino Hospital,

Bucharest, Romania

Background: Systemic sclerosis (SSc) is a connective tissue disease characterized by skin and internal organs fibrosis, microvascular impairment and frequently by disability and early retirement.

Objectives: To assess employment status, risk factors for early retirement (ER) and the associations of ER with disease characteristics and with patients' healthrelated questionnaires (Scleroderma Health Assessment Questionnaire (SHAQ)) and hand function (Duruoz hand index (DHI)).

Methods: This study included patients with SSc according to the 2013 ACR/EULAR classification criteria, examined in our EUSTAR center from 11.2011 to 11.2016, who were under the legal age of retirement of in our country $(62$ years). Patients completed a work assessment questionnaire, the $\mathrm{DHI}$ and the SHAQ, as well as a full assessment as per the recommendations of EUSTAR. Logistic regression was used to investigate the associations between employment status (outcome) and potential predictors (including socio-economic status, education, disease characteristics and health-related questionnaires).

Results: There were 66 patients ( 8 males, mean $\pm S D$ age $49.1 \pm 9.3$ years, 19 with diffuse cutaneous SSc (dcSSc), 46 with history of digital ulcers (DUs) and 23 with joint contractures) included. Forty-two patients lived in urban environments and 42 had higher education (high school or above).

Twenty patients were active professionally, whereas 46 were retired, of which 32 retired because of SSc. Of those active professionally, 8 had to do manual labor,

7 had to spend many hours at work standing and 3 had a cold or moist work environment.

Using logistic regression adjusted for age and gender, higher education was found to be highly associated with employment (OR (95\% Cl) $9.0(1.5,52.4)$ ), whereas labor conditions (manual labor, stress) had no significant influence on employment status in our cohort. No association was found between employment status and disease characteristics or SHAQ and DHI questionnaires.

Conclusions: SSc is associated with substantial work disability and unemployment. Completing less education than high school was associated with early retirement.

Acknowledgements: "This abstract was realized as part of the "Development of a computer-based nailfold videocapillaroscopy (NVC) system for longitudinal evaluation of patients with systemic sclerosis" (QUANTICAP) project, financed by the UEFIS-CDI PN-II-PT-PCCA-2013-4-1589 grant

Disclosure of Interest: None declared

DOI: 10.1136/annrheumdis-2017-eular.5892

\section{THU0608 ASSOCIATION BETWEEN ALLOPURINOL DOSE-TITRATION AND SERUM URIC ACID LEVELS IN GOUT PATIENTS: US ELECTRONIC HEALTH RECORD DATA}

A.-C. Fu ${ }^{1}$, D.C. Taylor ${ }^{2}$, D.S. Reasner ${ }^{2} .{ }^{1}$ Ironwood Pharmaceuticals, Inc. Cambridge, Ashmore and Cartier Islands; ${ }^{2}$ Ironwood Pharmaceuticals, Inc., Cambridge, United States

Background: Allopurinol is a first-line urate lowering therapy for patients with gout. The American College of Rheumatology guidelines recommend allopurinol dose-titration to maintain serum uric acid (sUA) levels $<6 \mathrm{mg} / \mathrm{dl}$. 